\title{
Real-world Evidence Needs Careful Interpretation
}

\author{
Peter Nash ${ }^{1}$ (D)
}

Determination plus persistence equals achievement.

Stanley T. Crawford

The Janus kinase (JAK) inhibitors have proven to be popular across the globe for an increasing variety of autoimmune inflammatory disorders seen in rheumatology, dermatology, and gastroenterology ${ }^{1}$. In patients with rheumatoid arthritis (RA), market share is on the rise in many countries, with 4 or $5 \mathrm{JAK}$ inhibitors available or under development, and most patient categories are comprehensively studied, such as methotrexate (MTX)-naïve, MTX-inadequate responders, biological disease-modifying antirheumatic drug (bDMARD)-inadequate responders, and monotherapy, in large randomized controlled trials (RCT) that have shown efficacy with a manageable safety profile.

It is clear, however, that the majority of patients with RA seen in clinical practice are ineligible for RCT. For example, a study of the German RABBIT registry found less than $35 \%$ of patients with RA would be eligible for an $\mathrm{RCT}^{2}$; therefore, real-world observational studies are important, particularly as these patients often have worse prognostic factors including more comorbidities, older age, longer disease duration, and increased number of prior DMARD use, and RCT may overestimate therapeutic effect $^{3}$. Further, large numbers of patients followed for long periods of time are required to show rare adverse effects.

Drug persistence over time is a surrogate for continued efficacy and the absence of AE leading to discontinuation, but there are important caveats. Drug persistence is affected by many variables such as polypharmacy, age, level of self-efficacy and social support, health perception and necessity belief, increased knowledge of RA, lower levels of education and income, as well as

$D r$ Nash received funding for research and honoraria for lectures and advice on behalf of Abbvie, Pfizer, Lilly, Novartis, Janssen, and Gilead.

${ }^{1} P$. Nash, MBBS(Hons) FRACP, School of Medicine, Griffith University, Queensland, Australia.

Address correspondence to Prof. P. Nash, Griffith University, Medicine, PO Box 308 Sunshine Coast, Nathan, Queensland 4111, Australia.

Email:drpnash@tpg.com.au. higher drug costs ${ }^{4}$. In observational studies, novel therapies are subject to selection bias - the necessity of drug failure before reimbursement, dosage restrictions mandated by regulators, time entering the market, persistence with therapy in partial responders when many other therapies have failed, efficacy as monotherapy in the MTX intolerant, the effect of competitors entering the market, as well as the ramifications of drug cost.

In this issue of The Journal, Fisher, $e t a^{5}$ have examined the persistence of JAK1/3 inhibitor tofacitinib in patients with RA compared to the persistence of bDMARD of a large, carefully performed, retrospective new user cohort study in a large Canadian MarketScan research database. Over a 4-year period, new users were compared and the time between treatment initiation to discontinuation or drug switch was determined. Further analysis examined post first-line therapy in patients switching to tofacitinib from a bDMARD. They concluded that new tofacitinib users had a shorter medication persistence (median 0.81 yrs) compared to bDMARD patients (median $1.02 \mathrm{yrs}$ ) with an HR of 1.14. However, they also concluded that patients who switch to tofacitinib from a bDMARD had the opposite effect, that is, longer persistence than patients who switched to a bDMARD (HR for discontinuation 0.90). Are these differences clinically significant? Further, shortcomings of the study include the lack of information on the causes of discontinuation as well as the percentage $(16 \%)$ of patients with non-RA diagnoses, from osteoarthritis to gout, psoriasis, ankylosing spondylitis, and vasculitis.

What is the clinician to make of such studies? How complete the data integrity of the Canadian Network for Observational Drug Effect Studies is and perhaps whether data entry is voluntary or mandated are relevant. The timing of tofacitinib entry to the Canadian market in relation to bDMARD and the provincial Canadian access regulations affect rheumatologist therapeutic choice and drug persistence as does, importantly, the size of the monotherapy market where JAK inhibitors and tocilizumab have an advantage over other bDMARD.

What have similar analyses shown? Two studies, ${ }^{6,7}$ found

See Tofacitinib persistence, page 16 
that tofacitinib was more commonly used as monotherapy than bDMARD, and persistence in treatment and adherence were quite comparable between tofacitinib and bDMARD. Another study showed patients initiating tofacitinib had longer disease duration and at the group level had been exposed to more bDMARD than patients initiating a bDMARD ${ }^{8}$. The Swiss Clinical Quality Management registry, which includes almost 2000 patients initiating treatment with tofacitinib, tumor necrosis factor inhibitors (TNFi), or non-TNFi bDMARD, found similar crude drug retention rates for the 3 cohorts ${ }^{9}$. After adjustment, a higher risk of discontinuation was associated with TNFi versus tofacitinib (HR 1.27). A higher number of prior bDMARD and greater BMI values were significantly associated with an increased risk of discontinuation. In contrast to the findings of Fisher, et al, an Australian study found the median persistence of treatment for the matched population was not significantly different at 33.8 months for patients prescribed with bDMARD and at 34.2 months for patients prescribed with tofacitinib; the reasons for discontinuation in the bDMARD and tofacitinib arms, respectively, were assessed as completion of treatment ( $33 \%$ vs $25 \%$ ), lack of efficacy ( $22 \%$ vs $17 \%$ ), secondary failure (16\% vs $10 \%)$, and adverse effects ( $16 \%$ vs $12 \%)^{10}$. More relevant is a Canadian study of long-term extension of clinical trials that showed median drug survival for all tofacitinib-treated patients was 4.9 years and estimated 2 - and 5-year drug survival rates were $75.5 \%$ and $49.4 \%$, respectively. Positive serology, low BMI, MTX monotherapy, or MTX dose $15 \mathrm{mg}$ or less per week, and absence of specific comorbidities (diabetes, hypertension, or cardiovascular disease) appeared to be associated with increased drug survival. The most common reasons for discontinuation were adverse effects (23.9\%), lack of patient willingness to participate $(10.1 \%)$, "other" reasons (6.2\%; i.e., any reason not otherwise classified), and lack/loss of efficacy $(3.6 \%)^{11}$.

In conclusion, real-world data are clinically important, and drug survival with appropriate caveats is a good surrogate for continued efficacy and lack of AE necessitating discontinuation. Studies such as those by Fisher, et al ${ }^{5}$ are informative but require cautious interpretation because findings are dependent on a variety of medical and nonmedical influences. Confirmation from similar studies in large registries from a variety of countries with differing medical systems would help to clarify the picture and advise on drug persistence of any novel therapy, in this instance the first JAK inhibitor in the rheumatology market.

\section{REFERENCES}

1. Nash P, Kerschbaumer A, Van der Heijde D, Smolen JS. AB0352 Consensus statement: use of Jakinib therapy in immune mediated inflammatory diseases [abstract]. Ann Rheum Dis 2020:79 Suppl 1:1475-6.

2. Zink A, Strangfeld A, Schneider M, Herzer P, Hierse F, Stoyanova-Scholz M. Effectiveness of tumor necrosis factor inhibitors in rheumatoid arthritis in an observational cohort study: comparison of patients according to their eligibility for major randomized clinical trials. Arthritis Rheum 2006;54:3399-407.

3. Kilcher G, Hummel N, Didden EM, Egger M, Reichenbach S; GetReal Work Package 4. Rheumatoid arthritis patients treated in trial and real world settings: comparison of randomized trials with registries. Rheumatology 2018;57:354-69.

4. Salt E, Frazier S. Adherence to disease modifying anti-rheumatic drugs in rheumatoid arthritis patients: a narrative review of the literature. Orthop Nurs 2010;29:260-75.

5. Fisher A, Hudson M, Platt RW, Dormuth CR; Canadian Network for Observational Drug Effect Studies Investigators. Tofacitinib persistence in patients with rheumatoid arthritis: a retrospective cohort study. J Rheumatol 2021;48:16-24.

6. Harnett J, Gerber R, Gruben D, Koenig AS, Chen C. Evaluation of real-world experience with tofacitinib compared with adalimumab, etanercept, and abatacept in RA patients with 1 previous biologic DMARD: data from a U.S. administrative claims database. J Manag Care Spec Pharm 2016; 22:1457-71.

7. Smith T, Harnett J, Gruben D, Chen C, Agarwal E, Woolcott J. Real-world experience with tofacitinib versus adalimumab and etanercept in biologic-naive patients with RA previously treated with methotrexate: data from a US administrative healthcare insurance claims database [abstract]. Arthritis Rheumatol 2017;69 Suppl 10:2831.

8. Caporali R, Zavaglia D. Real-world experience with tofacitinib for the treatment of rheumatoid arthritis. Clin Exp Rheumatol 2019;37:485-95.

9. Finckh A, Herzog L, Scherer A, Dudler J, Moeller B, Ciurea A; Physicians of the SCQM. Drug retention of tofacitinib versus biologic antirheumatic agents in rheumatoid arthritis: observational data from the Swiss SCQM registry [abstract]. Ann Rheum Dis 2017;76 Suppl 2:267.

10. Bird P, Littlejohn G, Butcher B, Smith T, da Fonseca Pereira $\mathrm{C}$, Witcombe D, et al. Real-world evaluation of effectiveness, persistence, and usage patterns of tofacitinib in treatment of rheumatoid arthritis in Australia. Clin Rheumatol 2020; 39:2545-51.

11. Pope JE, Keystone E, Jamal S, Wang L, Fallon L, Woolcott J, et al. Persistence of tofacitinib in the treatment of rheumatoid arthritis in open-label, long-term extension studies up to 9.5 years. ACR Open Rheumatol 2019;1:73-82. 